\title{
Comparison of Subcapsular and Total Orchiectomy in Patients with Prostate Cancer
}

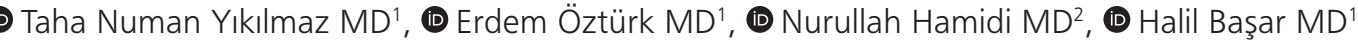 \\ 'University of Health Sciences, Dr. Abdurrahman Yurtaslan Ankara Oncology Training and Research Hospital, Clinic of Urology, Ankara, Turkey \\ ${ }^{2}$ Atatürk Training and Research Hospital, Clinic of Urology, Ankara, Turkey
}

\begin{abstract}
Objective: We aimed to compare the oncologic and functional outcomes of patients who underwent subcapsular or total orchiectomy for surgical castration in prostate cancer.

Materials and Methods: We studied 65 patients who underwent total or subcapsular orchiectomy with a diagnosis of prostate cancer between April 2014 and May 2017. At postoperative 3-month follow-up, prostate specific antigen (PSA) and total testosterone levels were measured and patients were asked about their psychological status due to organ loss. Results were compared between the groups.

Results: Sixty-five patients were evaluable: 23 had subcapsular and 42 had total orchiectomy. The mean age of the cases was 71.4 (60-83) years, the mean PSA level was $45.4 \mathrm{ng} / \mathrm{dL}(4-3800 \mathrm{ng} / \mathrm{dL})$, and 41 cases had metastatic foci. There was no significant difference between the complication rates of the groups, but duration of the operation was significantly shorter in the subcapsular orchiectomy group. The two groups had similar mean PSA and testosterone levels at postoperative 3 months, but significantly more patients with total orchiectomy reported psychological problems due to organ loss.

Conclusion: Subcapsular orchiectomy should be preferred for surgical castration because of the short duration of operation and the advantage of organ presence within the scrotal sac after surgery. In terms of oncologic outcomes, subcapsular orchiectomy shows no difference from total orchiectomy and is a safe alternative.
\end{abstract}

Keywords: Subcapsular orchiectomy, total orchiectomy, prostate cancer

\section{Introduction}

Prostate cancer is the second most common malignant neoplasm in men after skin cancer. It accounts for $28 \%$ of all cancers in males. Although the introduction of the prostate-specific antigen (PSA) test in the 1960s has facilitated the diagnosis of prostate cancer, $5-10 \%$ of these patients are diagnosed with distant metastasis (1). Diethylstilbestrol treatment was first prescribed after it was discovered that prostate cancer is an androgen-dependent condition. Huggins and Hodges (2) first described total orchiectomy in 1941, claiming that orchiectomy and estrogen were equally effective in metastatic cases. This treatment was shown to provide 18-34 months of progression- free survival in $90 \%$ of patients (3). While gonadotropin-releasing hormone $(\mathrm{GnRH})$ agonists have been used since the 1980s for chemical castration, $\mathrm{GnRH}$ antagonists have also been used in recent years (4). Antiandrogen therapies are used to prevent the "flare phenomenon" that may occur with GnRH agonists, but their use for maximal androgen blockade is much less common today.

Surgical castration is preferred due to the late onset of effect and high cost of medical castration. The only reason explaining the avoidance of surgical castration is shown to be the psychological distress that can result from having an empty scrotum (1). With this in mind, Riba (5) first described subcapsular orchiectomy

Address for Correspondence: Taha Numan Yıkılmaz MD, University of Health Sciences, Dr. Abdurrahman Yurtaslan Ankara Oncology Training and Research Hospital, Clinic of Urology, Ankara, Turkey Phone: +90 3123360909 E-mail: numanyikilmaz@gmail.com ORCID-ID: orcid.org/0000-0001-8410-2474 Received: 02.10.2017 Accepted: 13.12.2017 
in 1942. In this technique, the outer wall of the tunica albuginea is preserved and a palpable mass is left after the operation to prevent the empty scrotum sensation. Several other techniques have been described to address this issue, such as inserting testicular prostheses, fat injection following total orchiectomy, and subepididymal orchiectomy, but none has been as successful as the simple subcapsular orchiectomy. In this study, we compared oncological follow-up results and the impact of surgery type in patients who underwent subcapsular and total orchiectomy in our clinic due to advanced or metastatic prostate cancer.

\section{Materials and Methods}

Patients who were scheduled for surgical castration due to prostate cancer and underwent total or subcapsular orchiectomy in our clinic between April 2014 and May 2017 were evaluated. Of the 83 patients who underwent orchiectomy during the study period, 18 were excluded due to missing data or incomplete follow-up. Thus, the data of 65 patients were analyzed in this retrospective study. The patients' demographic characteristics, comorbidities, preoperative metastatic status, treatments received, surgical anesthesia risks, surgery time, length of hospital stay, drainage volumes, and complications were recorded. All patients included in the study provided informed consent for the operation. Because the study was retrospective, ethics committee approval was not obtained. The study was designed in accordance with the Declaration of Helsinki.

In the subcapsular orchiectomy technique practiced in our clinic, we use a no. 15 scalpel to create a single incision through the skin, subcutaneous layer, tunica vaginalis, and tunica albuginea to access the testicular tissue. Perforating the tunica albuginea allows the testicular parenchyma to be extruded through this small incision. The testicular tissue is then peeled from the tunica albuginea using wet gauze. Hemostasis is achieved with cauterization and the tunica albuginea is closed with 3-0 vicryl hemostatic suture. The skin is closed with interrupted 2-0 vicryl sutures without placing a Penrose drain and "turban" compression dressing is applied to the scrotum. We perform the standard total orchiectomy technique, wherein an incision is made in the skin and subcutaneous tissue, then the tunica vaginalis is separated from the subcutaneous tissue via blunt dissection and the testis is removed together with the tunica vaginalis. Both procedures were performed under regional anesthesia and all patients were discharged within 48 hours postoperatively.

At postoperative 3-month follow-up, the patients' PSA and total testosterone levels were measured and they were asked about their psychological state regarding organ loss. Results of the two groups were compared. Statistical analysis were performed using SPSS Windows 21.0 software package and the Mann-Whitney $\mathrm{U}$ test. $\mathrm{P}<0.05$ was accepted as the minimum significance level.

\section{Results}

Eighty-three patients were initially selected for the study, but only 65 patients met the inclusion criteria. Four patients were excluded because testicular prostheses were inserted during surgery, 3 patients died in the early postoperative period, 2 patients had a history of previous scrotal surgery, and 9 patients were lost to follow-up. Twenty-three of the remaining patients underwent subcapsular orchiectomy and 42 underwent total orchiectomy. Choice of surgical procedure was based on the surgeon's preference. Forty-one of the 65 patients who underwent surgical castration had been diagnosed with metastatic prostate cancer prior to surgery. In 12 of these patients, surgical castration was performed as the first treatment option without attempting chemical castration due to the patient's age, preference, socioeconomic and cultural level, and metastatic burden, as well as the doctor's preference. Surgical castration was not chosen as primary treatment for any of the non-metastatic patients. The patients' mean age was 71.4 years (60-83 years) and their mean preoperative PSA level was $45.4 \mathrm{ng} / \mathrm{dL}(4-3800 \mathrm{ng} / \mathrm{dL})$. In the same surgical session, 17 patients underwent transurethral resection of the prostate (TURP) due to lower urinary system complaints, and 1 patient also had penile prosthesis implantation. Patients who underwent subcapsular orchiectomy were included in group 1 , and patients who underwent total orchiectomy in group 2. Differences between the groups in terms of demographic characteristics and complications are summarized in Table 1. Preoperative PSA levels were significantly lower in patients who underwent subcapsular orchiectomy compared to patients who had total orchiectomy ( $21.6 \mathrm{ng} / \mathrm{dL}$ and $49 \mathrm{ng} / \mathrm{dL}$, respectively). There were no significant differences between the groups in terms of age or American Society of Anesthesiologists physical status scores. There was also no significant difference between the mean surgery times of the groups, though the surgery was slightly shorter in group 1 (23 min and $32 \mathrm{~min}$, respectively). TURP and prosthesis implantation procedures performed simultaneously with orchiectomy were not included when calculating surgery times. Patients in group 1 did not require drain placement; exudate saturated an average of 1.5 sponge dressings. In group 2, a Penrose drain was inserted for 27 of the patients and left

Table 1. Comparison of demographic data and characteristics between groups

\begin{tabular}{|l|l|l|l|}
\hline & $\begin{array}{l}\text { Group 1 } \\
\text { Subcapsular } \\
\text { orchiectomy } \\
\mathbf{n = 2 3}\end{array}$ & $\begin{array}{l}\text { Group 2 } \\
\text { Total } \\
\text { orchiectomy } \\
\mathbf{n = 4 2}\end{array}$ & $\mathbf{p}$ \\
\hline Age (years) & 70.2 & 71.8 & 0.2 \\
\hline PSA (ng/dL) & 21.6 & 49.2 & 0.03 \\
\hline ASA & 2.7 & 2.6 & 0.8 \\
\hline Surgery duration (min) & 23 & 32 & 0.08 \\
\hline Same-day discharge (\%) & $56 \%$ & $15 \%$ & 0.005 \\
\hline TURP & 7 & 10 & - \\
\hline Complication rate (\%) & 9 & 25 & 0.2 \\
\hline Postoperative PSA (ng/dL) & 3.1 & 4.1 & 0.6 \\
\hline $\begin{array}{l}\text { Postoperative } \\
\text { testosterone (ng/mL) }\end{array}$ & 29 & 33 & 0.8 \\
\hline Satisfaction rate (\%) & 81 & 40 & 0.001 \\
\hline $\begin{array}{l}\text { ASA: American Society of Anesthesiologists, PSA: Prostate specific antigen, TURP: } \\
\text { Transurethral resection of the prostate }\end{array}$ \\
\hline
\end{tabular}


in place for at least 24 hours postoperatively. As exact drainage volumes could not be calculated in both groups, the difference between groups could not be assessed. When patients who did not undergo simultaneous TURP were compared in terms of time to discharge, it was found that 9 of the patients in group 1 (56\%) and 5 of the patients in group 2 (15\%) were discharged on the same day as surgery. There was no significant difference between the two groups in length of hospital stay. Complications were observed in a total of 13 patients from both groups and 4 patients required revision surgery. These patients underwent wound revision, and there was no significant difference between the groups. PSA and total testosterone levels analyzed at postoperative 3 months did not differ significantly between the groups. At postoperative 3-month follow-up, the patients were asked to rate their satisfaction with the operation and responses were recorded as "satisfied" or "not satisfied". Eighty-one percent of the patients in group 1 were happy due to the sensation of having testes, while only $40 \%$ of the patients in group 2 expressed satisfaction; this difference was statistically significant.

\section{Discussion}

Antiandrogen therapy for metastatic prostate cancer can be achieved through chemical or surgical castration. Surgical castration is performed through a scrotal incision as either total orchiectomy or as subepididymal or subcapsular orchiectomy, which gives the patient the feeling that the scrotum is partially full. As the testosterone-producing parenchyma is removed in all three techniques, they provide equivalent treatment efficacy (6). Although surgical castration is preferred as an inexpensive and easy procedure, its biggest demonstrable disadvantages include complications associated with surgical procedures and the psychological trauma of the "empty scrotum" feeling (1). In the present study, we observed no marked difference in terms of complications between the patients who underwent subcapsular orchiectomy and total orchiectomy, and there were no life-threatening complications. In their study of 74 patients, Zhang et al. (7) reported complications rates of 3\% and $22 \%$ respectively for patients undergoing subcapsular and total orchiectomy, and similar rates have been confirmed in numerous other studies $(8,9)$. These studies have generally shown that the subcapsular technique has fewer complications. Although we observed a similar ratio in our study, the difference was not statistically significant, which may be related to the fact that the patients were operated by different surgeons. The need for revision surgery was quite low in our patient group (6\%), consistent with the literature.

A notable finding of our study was the short surgery times in patients undergoing subcapsular orchiectomy, which was not consistent with the literature. Roosen et al. (8) reported that the subcapsular technique took significantly longer to perform. We attribute this discrepancy to our variation on the surgical technique. In our technique, the testicular parenchyma is accessed and removed via a single full-thickness incision and the layers are closed as a single piece. In contrast, Roosen perform this procedure by opening the layers one by one.

In accordance with the literature, we had success with our sameday discharge surgeries using the subcapsular technique. Not placing drains after surgery considerably shortens the patient's stay in hospital. This clearly demonstrates the economic advantage of this method.

There was no difference between the groups in terms of oncological outcomes at postoperative 3 months. In the literature, it is argued that both techniques are oncologically successful when appropriate surgical procedures are followed $(7,8,10,11)$. High testosterone level despite orchiectomy is linked to adrenal production and metastatic foci.

In the present study, we investigated the postoperative "empty scrotum" feeling, which has been discussed the literature but patients are not usually asked about. Patients in our study were asked during follow-up whether they experienced this distress, and we found that $19 \%$ of patients who underwent the subcapsular technique and $60 \%$ of those who underwent the total technique were not psychologically satisfied with the procedure. In other studies comparing these techniques, psychological problems are mentioned but the patients were not asked to rate their satisfaction $(1,8)$.

\section{Study Limitations}

Limitations of this study include its retrospective design and the small number of patients. Furthermore, the same surgeon did not perform all of the procedures, and surgery times were difficult to calculate because some patients had additional procedures (TURP, prosthesis implantation). The patients' preferences were another source of irregularity.

\section{Conclusion}

At our clinic, we prefer to implement a subcapsular technique that is modified from that described in the literature. Our variation on the subcapsular technique is advantageous in terms of its shorter surgery time and same-day discharge due to the use of regional anesthesia and the unnecessity of drains. The modified subcapsular technique is also superior to the total orchiectomy technique in terms of complications and the need for revision surgery while yielding equivalent oncological results. Most importantly, the subcapsular technique provides greater patient satisfaction after surgery due to the feeling of a full scrotum.

\section{Ethics}

Ethics Committee Approval: Because the study was retrospective, ethics committee approval was not obtained. The study was designed in accordance with the Declaration of Helsinki.

Informed Consent: All patients included in the study provided informed consent for the operation.

Peer-review: Externally and internally peer-reviewed.

\section{Authorship Contributions}

Surgical and Medical Practices: T.N.Y., H.B., Concept: T.N.Y., E.Ö., Design: T.N.Y., Data Collection or Processing: T.N.Y., N.H., Analysis or Interpretation: T.N.Y., H.B., Literature Search: E.Ö., N.H., Writing: T.N.Y.

Conflict of Interest: No conflict of interest was declared by the authors.

Financial Disclosure: The authors declared that this study received no financial support. 


\section{References}

1. Rud O, Peter J, Kheyri R, et al. Subcapsular orchiectomy in the primary therapy of patients with bone metastasis in advanced prostate cancer: an anachronistic intervention? Adv Urol 2012;2012:190624.

2. Huggins C, Hodges CU. Studies on prostatic cancer; effect of castration, of estrogenand of androsterone injection onthe serum phosphatases in metastatic carcinoma of the prostate. Cancer Res $1941 ; 1: 293$.

3. Lam JS, Leppert JT, Vemulapalli SN, et al. Secondary hormonal therapy for advanced prostate cancer. J Urol 2006;175:27-34.

4. Dellis A, Papatsoris A. Therapeutic outcomes of the LHRH antagonists. Expert Rev Pharmacoecon Outcomes Res. 2017;17:481-488.

5. Riba LW. Subcapsular castration for carcinoma of prostate. J Urol 1942;48:384-387.

6. Østergren PB, Kistorp C, Fode M, et al. Luteinizing Hormone-Releasing Hormone Agonists are Superior to Subcapsular Orchiectomy in
Lowering Testosterone Levels of Men with Prostate Cancer: Results from a Randomized Clinical Trial. J Urol 2017;197:1441-1447.

7. Zhang XZ, Donovan MP, Williams BT, Mohler JL. Comparison of subcapsular and total orchiectomy for treatment of metastatic prostate cancer. Urology 1996;47:402-404.

8. Roosen JU, Klarskov OP, Mogensen P. Subcapsular versus total orchiectomy in the treatment of advanced prostate cancer: a randomized trial. Scand J Urol Nephrol 2005;39:464-467.

9. Desmond AD, Arnold AJ, Hastie KJ. Subcapsular orchiectomy under local anaesthesia. Technique, results and implications. $\mathrm{Br} J$ Urol 1988;61:143-145.

10. Vickers MA Jr, Lamontagne DP, Guru KA, et al. Autologous tunica vaginalis and subcapsular orchiectomy: a hormonal therapy for prostate cancer. J Androl 2004;25:375-381.

11. Chapman JP. Comparison of testosterone and LH values in subcapsular vs total orchiectomy patients. Urology 1987;30:27-28. 